\title{
Cognitive profile of subcortical ischaemic vascular disease
}

\author{
H Jokinen, H Kalska, R Mäntylä, T Pohjasvaara, R Ylikoski, M Hietanen, O Salonen, M Kaste, \\ T Erkinjuntti
}

J Neurol Neurosurg Psychiatry 2006;77:28-33. doi: 10.1136/jnnp.2005.069120

See end of article for authors' affiliations

Correspondence to: Hanna Jokinen,

Department of Neurology Helsinki University Central Hospital, PO Box 302 , FIN-00029 HUS, Helsinki, Finland; hanna.jokinen@ helsinki.fi

Received 5 April 2005

Revised version received

1 June 2005

Accepted 14 June 2005

\begin{abstract}
Objectives: Subcortical ischaemic vascular disease (SIVD) is a subtype of vascular cognitive impairment characterised by extensive white matter lesions and multiple lacunar infarcts. Radiologically defined diagnostic criteria for SIVD have been introduced, but only a few studies have presented empirical data on its clinical and cognitive features. The aim of this study is to describe in detail the neuropsychological characteristics of patients with SIVD from a large well defined stroke cohort.

Methods: A sample of 323 consecutive patients with ischaemic stroke, aged 55-85 years, was investigated using neuropsychological examination and magnetic resonance imaging (MRI). Patients fulfilling the MRI criteria of SIVD $(n=85)$ were compared to the other stroke patients $(n=238)$ and to normal control subjects $(n=38)$.

Results: Cognitive performance of the SIVD group was inferior to that of the normal control group throughout all domains. As compared to the other stroke patients, the SIVD group performed significantly worse in tests measuring executive functions and delayed memory recall. Adjusting for depression had no effect on these results. Instead, after controlling for medial temporal lobe atrophy, the differences disappeared for delayed memory but remained significant for executive functions.

Conclusion: Executive deficits are the most prominent cognitive characteristic associated with SIVD. Patients with SIVD also exhibit subtle deficits in delayed memory, which is explained in part by medial temporal lobe atrophy. Cognitive and mood changes seem to be parallel but independent processes related to SIVD. The results support the concept of SIVD as a separate clinical entity.
\end{abstract}

$\mathrm{T}$ he concept of vascular cognitive impairment ${ }^{1}$ covers a wide spectrum of cognitive dysfunction ranging from subtle and clinically often undetected deficits to overt dementia. The major subtypes of vascular dementia are cortical vascular dementia, strategic infarct dementia, and subcortical vascular dementia, which all have varying aetiological mechanisms and manifestations. ${ }^{2}$ Because of the heterogeneity of the clinical appearance of vascular cognitive impairment and dementia, the concept of subcortical ischaemic vascular disease (SIVD), defined by specific features on magnetic resonance imaging (MRI), has been adopted. ${ }^{2}$ SIVD is regarded as a relatively homogeneous subgroup of vascular cognitive impairment with a more cohesive aetiology and a more predictable outcome. It arises from small vessel disease and is characterised by extensive cerebral white matter lesions and lacunar infarcts in deep grey and white matter structures. ${ }^{3}$ Diagnostic consensus criteria have been introduced recently. ${ }^{4}$ However, empirical data describing its clinical and cognitive manifestations are still sparse.

A previous study by our group ${ }^{5}$ has shown that patients with SIVD, as compared to other elderly stroke patients, more often have a history of progressive cognitive decline, dependence in activities of daily living, executive dysfunction, and DSM-III defined dementia. Other studies investigating the cognitive characteristics of patients with SIVD are few and have used diverse diagnostic criteria. A recent study has found subtle declines in executive functions and visual memory in non-demented patients with one or more subcortical lacunes. ${ }^{6}$ It has been suggested that subcortical ischaemic vascular pathology can be distinguished from Alzheimer's disease by neuropsychological features, that is, better recognition memory ${ }^{7}$ and spatial cognition ${ }^{7}$ but worse verbal fluency. ${ }^{8}$ Due to a disruption of the frontal-subcortical circuits, the frontal lobe mediated executive functions are considered particularly vulnerable to ischaemic lesions. ${ }^{9-11}$
The aim of this study was to describe in detail the neuropsychological profile of SIVD in order to further validate the brain imaging criteria in use. In our large consecutive stroke sample, we compared patients fulfilling the criteria of SIVD to all the other stroke patients (OS) and to normal control subjects (NC). All subjects underwent a comprehensive neuropsychological examination and MRI.

\section{METHODS}

\section{Subjects and study protocol}

As part of the Helsinki Stroke Aging Memory (SAM) Study, 486 consecutive patients, aged $55-85$ years, were examined 3 months after an ischaemic stroke. The examination included a clinical neurological assessment, brain MRI, and a detailed neuropsychological assessment. The study protocol and the clinical characteristics of the cohort have been described in previous publications. ${ }^{512}{ }^{13}$ In brief, 409 patients participated in the neuropsychological assessment after patients with considerable hearing or sight disabilities, or reduced level of consciousness, and those who were unwilling to participate were excluded. Cases with inadequate neuropsychological or MRI data $(\mathrm{n}=86)$ were also excluded from this study. The total patient sample was therefore 323 subjects. Compared to the included patients, the excluded patients $(n=163)$ were older $(72.9 \vee 70.3$ years, $\mathrm{p}<0.001)$,

Abbreviations: ${ }^{B^{*}}$, significant result after Bonferroni correction; $\eta_{p}{ }^{2}$, partial eta squared; DSM-III-R, Diagnostic and Statistical Manual for Mental Disorders-Revised; DWMH, deep white matter hyperintensities; FOME, Fuld Object Memory Evaluation; MANCOVA, multivariate analysis of covariance; MRI, magnetic resonance imaging; MTA, medial temporal lobe atrophy; NC, normal control subjects; OS, other stroke; $\mathrm{PVH}$, periventricular white matter hyperintensities; SD, standard deviation; SIVD, subcortical ischaemic vascular disease; WAIS-R, Wechsler Adult Intelligence Scale-Revised; WCST, Modified Wisconsin Card Sorting Test; WMS, Wechsler Memory Scale; WMS-R, Wechsler Memory Scale-Revised 
had less education ( $8.4 v 9.5$ years, $\mathrm{p}<0.01)$, and more severe stroke as measured with the Scandinavian Stroke Scale (46.0 $v 55.5, \mathrm{p}<0.001)$. They were also more impaired in activities of daily living according to the Barthel Index (14.8 $v$ 19.0, $\mathrm{p}<0.001)$ and had lower global cognitive status in the MiniMental State Examination ${ }^{14}(22.1 \vee 26.3, \mathrm{p}<0.001)$. There were no gender differences between the excluded and included patients.

The control subjects $(n=38)$ were drawn from a population based study conducted previously. ${ }^{15}$ They were clinically evaluated as neurologically healthy and all participated in the neuropsychological assessment and MRI.

The study was approved by the Ethics Committee of the Department of Neurology, Helsinki University Central Hospital. The study design was fully explained to the subjects. Those who participated gave their informed consent.

\section{Neuropsychological assessment}

The neuropsychological battery covered a wide range of cognitive domains, and standard test instructions and scoring were used. ${ }^{16}{ }^{17}$ In order to reduce the number of variables, seven composite scores were constituted by calculating the mean of the standardised $\mathrm{z}$ scores across the whole study sample in each domain. The distributions of the individual and composite scores were approaching normality. When appropriate, the scales were first reversed to correspond with each other. The variables and the reliability coefficients for the composite scores are presented in table 1.

Speed of mental processing was assessed with the Trail Making test part $\mathrm{A}^{18}$ and a short version of the Stroop test, coloured dots section (Stroop dots). ${ }^{19}$ The total time to complete the tasks was measured.

Table 1 Neuropsychological variables used in composite $\mathrm{z}$ scores

\begin{tabular}{ll}
\hline Cognitive domains and variables & $\begin{array}{l}\text { Reliability } \\
\text { coefficient } \\
\text { (Cronbach's } \alpha \text { ) }\end{array}$ \\
\hline $\begin{array}{l}\text { Speed of mental processing } \\
\text { Trail Making A test, time }\end{array}$ & 0.66 \\
Stroop dots, time & \\
Executive functions & 0.78 \\
Trail Making test, subtraction score (B time-A time) & \\
Trail Making B test, correct responses & \\
Stroop test, subtraction score (words, time-dots, time) \\
Stroop words, correct responses \\
WCST, correct responses \\
WCST, perseverations \\
Verbal fluency, animal category \\
Verbal fluency, letter word list generation \\
Short term memory (working memory) \\
WMS digit span, forwards \\
WMS digit span, backwards \\
Immediate recall \\
WMS-R logical memory story A, immediate recall \\
WMS-R visual reproduction, immediate recall \\
FOME, total retrieval \\
Delayed recall \\
WMS-R logical memory story A, delayed recall \\
WMS-R visual reproduction, delayed recall \\
FOME, delayed recall \\
Verbal intellectual functions \\
WAIS-R similarities \\
WASS-R information, shortened version \\
WAIS-R comprehension, shortened version \\
Visuospatial functions \\
WAIS-R block design \\
\hline
\end{tabular}

The reliability coefficient for the visuospatial score was not calculated as it contained only one test.

FOME, Fuld Object Memory Evaluation; WAIS-R, Wechsler Adult Intelligence Scale; WCST, Modified Wisconsin Card Sorting Test; WMS, Wechsler Memory Scale; WMS-R, Wechsler Memory Scale-Revised
Executive functions were evaluated with the Trail Making test part $\mathrm{B}^{18}$ and the Stroop coloured colour names section (Stroop words) ${ }^{19}$ as measuring mental flexibility, set shifting, and response inhibition. In order to extract the executive component in these tests, a difference score was calculated by subtracting the time taken in Trail Making A from the time taken in Trail Making B. A similar calculation was performed for the Stroop dots and words tests. The number of correct responses was also recorded for the Trail Making B and the Stroop words tests. Abstract problem solving and the ability to shift cognitive sets were assessed with the modified version of the Wisconsin Card Sorting Test (WCST), ${ }^{20}$ where the parameters were the number of correct responses and perseverations. Verbal fluency was assessed in semantic category (animals) and letter word list generation (words beginning with the letter $\mathrm{K}$ ) in $60 \mathrm{~s}^{21}$

Short term memory (working memory) was measured with the digit span forward and backward subtests of the Wechsler Memory Scale (WMS), ${ }^{22}$ in which the scores were the longest correctly repeated series of digits.

Immediate memory was evaluated by using the Fuld Object Memory Evaluation (FOME, total retrieval of five learning trials), ${ }^{23}$ and the immediate recalls of the logical memory (story A) and visual reproduction subtests of the Wechsler Memory Scale-Revised (WMS-R). ${ }^{24}$

Delayed memory was assessed with the delayed free recalls of the FOME, and WMS-R logical memory and visual reproduction subtests.

Verbal intellectual functions were evaluated with the similarities subtest, and shortened versions of the information (10 items) and comprehension (four items) subtests of the Wechsler Adult Intelligence Scale-Revised (WAIS-R). ${ }^{25}$

Visuospatial functions were assessed with the block design subtest of the WAIS-R. ${ }^{25}$

\section{Magnetic resonance imaging}

The radiological method has been described in previous reports. ${ }^{5627}$ MRI was performed with a superconducting $1.0 \mathrm{~T}$ system and the images were reviewed by a neuroradiologist (RM) blinded to the clinical data. Focal lesions were recorded according to their number, location, side, and type. Lesions approaching the signal characteristics of cerebrospinal fluid on Tl weighted images and measuring over $3 \mathrm{~mm}$ in diameter were regarded as brain infarcts. Infarcts were classified by size into four groups according to largest diameter, and the average radii were used for volume calculations. The infarct was determined as lacunar if it was situated in deep white or grey matter, was 3-9 $\mathrm{mm}$ in diameter, and was supplied by the deep branches of the anterior, middle, or posterior cerebral arteries, or the internal carotid artery.

White matter hyperintensities were assessed on proton density weighted images in periventricular ( $\mathrm{PVH})$ and in deep, borderzone, and subcortical regions (deep white matter hyperintensities, DWMH), and were graded from 0 to 3 as detailed earlier. ${ }^{26}{ }^{27}$ Brain atrophy was rated visually from 0 to 3 separately on both hemispheres based on Tl weighted images. General cortical atrophy was rated in the frontal, parietal, and occipital lobes, and in the temporal neocortex. Medial temporal lobe atrophy (MTA) was rated on coronal slices in the hippocampal formation and the entorhinal cortex comprising the parahippocampal gyrus. Central atrophy was rated by assessing the width of the temporal, frontal, and occipital horns, the bodies of lateral ventricles, and the third ventricle. The ratings of cortical and central atrophy and MTA were combined into three sum variables for the purposes of the present study (ranges 0-24, 0-27, and $0-12$, respectively). ${ }^{28}$ 


\section{Definition of SIVD}

SIVD was determined according to the MRI criteria (for full details, see Erkinjuntti $e a^{4}$ ) that include (a) predominantly white matter cases: extending periventricular and deep white matter lesions, and (b) predominantly lacunar cases: multiple lacunes $(>5)$ in deep grey matter and at least moderate white matter lesions. Cases with cortical and/or corticosubcortical non-lacunar territorial infarcts, borderzone infarcts, haemorrhages, signs of normal pressure hydrocephalus, and specific causes of white matter lesions were excluded from SIVD. All the other patients not fulfilling these criteria were classed as "other stroke patients". Patients with both first ever strokes and with previous stroke incidents were included.

\section{Data analysis}

The demographic and clinical characteristics of the study groups were examined with independent-samples $t$ test, analysis of variance, and $\chi^{2}$ test. Neuropsychological test performance was studied with a multivariate analysis of covariance (MANCOVA) of the composite z scores and with univariate analyses of covariance of the raw scores. Pairwise contrasts were calculated between the covariate adjusted group means by comparing the SIVD group to the OS and NC groups. A p level $<0.05$ was regarded as statistically significant, but for the pairwise contrasts the more conservative Bonferroni correction is also reported (0.05/ $2=0.025$; denoted in the text with ${ }^{\mathrm{B}^{*}}$, if significant). Effect sizes for the group differences were analysed with partial eta squared $\left(\eta_{\mathrm{p}}{ }^{2}\right)$ reflecting the proportion of the total variance attributable to the effect; it is considered small if 0.01 , moderate around 0.06 , and high if $>0.13$ (converted from $\mathrm{f}$ according to Cohen). ${ }^{29}$

\section{RESULTS}

\section{Demographic and clinical characteristics}

Of the 323 patients, 85 fulfilled the criteria for SIVD. The characteristics of the study groups are presented in table 2 ( see also Pohjasvaara et $a l^{5}$ ). As to the demographic variables, the study groups differed significantly in terms of age and education but not gender. Pairwise contrast comparisons revealed significant differences between the patients with SIVD and OS in age $(\mathrm{p}<0.05)$ and education $\left(\mathrm{p}<0.0 \mathrm{1}^{\mathrm{B}^{*}}\right)$. The SIVD group differed from the NC group in age $\left(\mathrm{p}<0.01^{\mathrm{B}^{*}}\right)$ but not education $(p=0.19)$. In the Mini-Mental State Examination, ${ }^{14}$ the SIVD group had lower scores than the NC group $\left(p<0.001^{B^{*}}\right)$, but the SIVD and OS groups did not differ from each other $(p=0.07)$. The presence of dementia according to the DSM-III-R criteria ${ }^{30}$ did not differentiate the two patient groups. However, patients with SIVD exhibited significantly more depressive symptoms than patients with OS as measured with the Beck Depression Inventory ${ }^{31}$ (21 items) (table 2).

In addition to the number and volume of the infarcts and white matter hyperintensities, the study groups differed from each other in terms of several other MRI findings (table 2). In contrast to the NC group, the patients with SIVD had significantly more cortical $\left(\mathrm{p}<0.001^{\mathrm{B}^{*}}\right)$ and central atrophy $\left(\mathrm{p}<0.00 \mathrm{I}^{\mathrm{B}^{*}}\right)$, and MTA $\left(\mathrm{p}<0.00 \mathrm{I}^{\mathrm{B}^{*}}\right)$. As compared to the OS group, they had more MTA $\left(\mathrm{p}<0.05^{\mathrm{B}^{*}}\right)$ but not more cortical $(\mathrm{p}=0.07)$ or central $(\mathrm{p}=0.10)$ atrophy.

\section{Neuropsychological performance of the study groups}

When the composite $\mathrm{z}$ scores of the neuropsychological tests were used as dependent variables, and age and education were used as covariates, the multivariate main effect for the study groups was highly significant (MANCOVA Wilks' $\lambda$ $\left.F=4.1, \mathrm{p}<0.0001, \eta_{\mathrm{p}}{ }^{2}=0.077\right)$. The study groups differed significantly from each other in all of the dependent variables (table 3). Pairwise comparisons showed that the SIVD group differed from the $\mathrm{NC}$ group in each composite $\mathrm{z}$ score. Moreover, the composite $\mathrm{z}$ scores for executive functions and delayed memory recall were significantly lower in patients with SIVD than in patients with OS. Between these groups, no significant differences were found in speed of mental processing, short term memory, immediate recall, verbal intellectual functions, or visuospatial functions. Figure 1 shows the neuropsychological test performance profile in patients with SIVD and OS as normalised in relation to the NC group. The results of the two patient groups remained unchanged even after controlling for the total score of the Beck Depression Inventory. Instead, when MTA was controlled, the patient groups still differed in executive functions $\left(\mathrm{p}<0.05, \eta_{\mathrm{p}}{ }^{2}=0.013\right)$ but not in delayed memory.

The results were also analysed by using the raw scores of the neuropsychological tests. Pairwise comparisons between the SIVD and OS groups, as calculated with the analysis of covariance adjusted for age and education, revealed

Table 2 Characteristics of the study groups

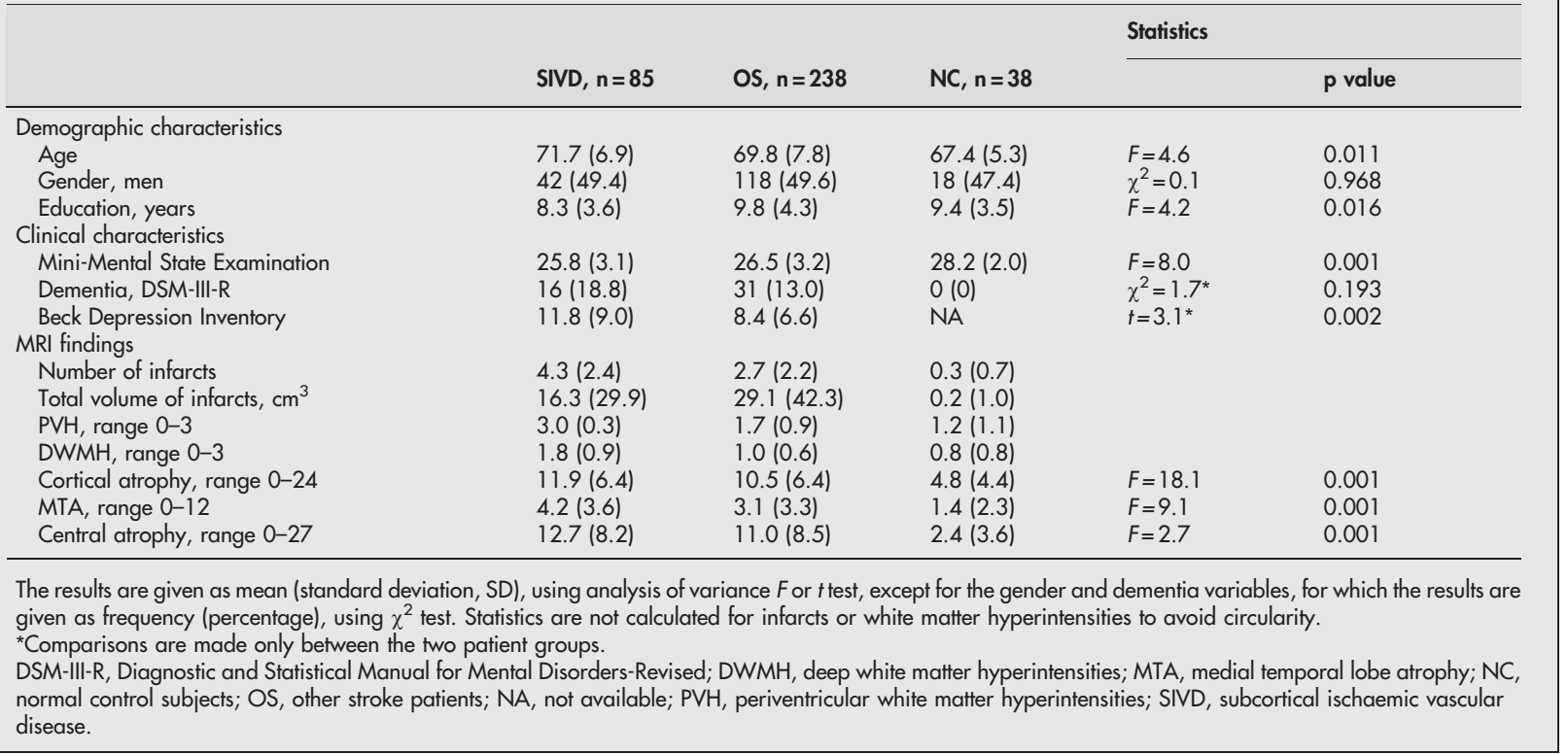




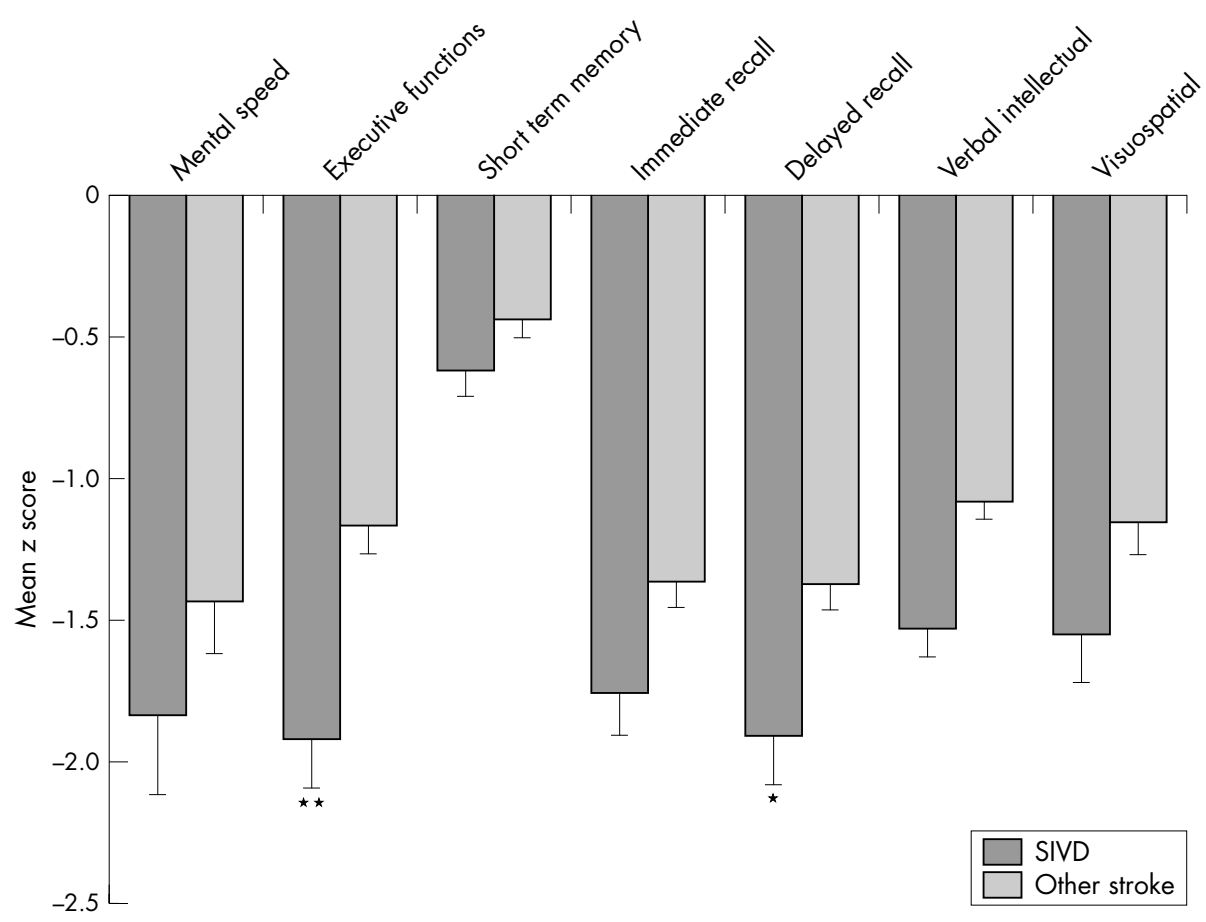

Figure 1 Neuropsychological test performance of patients with subcortical ischaemic vascular disease (SIVD) and other stroke patients standardised according to the normal control group. Error bars indicate standard error. ${ }^{*} \mathrm{p}<0.05,{ }^{* *} \mathrm{p}<0.01$.

significant differences in the delayed recall of FOME ( SIVD mean 7.1 (SD 2.2) $v$ OS mean 7.9 (SD 1.9), $\mathrm{p}<0.01^{\mathrm{B}^{*}}$, $\left.\eta_{\mathrm{p}}^{2}=0.020\right)$, verbal fluency, animal category $(14.3(6.0) v$ $\left.17.2(6.2), \mathrm{p}<0.01^{\mathrm{B}^{*}}, \eta_{\mathrm{p}}{ }^{2}=0.024\right)$ and letter word list generation (9.6 (5.2) $v 11.7(5.6), \mathrm{p}<0.05^{\mathrm{B}^{*}}, \eta_{\mathrm{p}}{ }^{2}=0.014$ ), as well as in the subtraction scores of the Trail Making test (147.5 (101.4) $v 117.7$ (75.9), $\left.\mathrm{p}<0.05, \eta_{\mathrm{p}}{ }^{2}=0.015\right)$ and the Stroop test $\left(35.0(28.1) \vee 27.2(19.6), \mathrm{p}<0.05^{\mathrm{B}^{*}}, \eta_{\mathrm{p}}{ }^{2}=0.019\right)$.

\section{DISCUSSION}

In order to characterise the cognitive deficits associated with SIVD, a large sample of elderly stroke patients and healthy controls were investigated using a detailed neuropsychological battery and MRI. The patients with SIVD tended to be older and had less education than the OS patients. After controlling for these demographic factors, the results revealed both quantitative and qualitative differences in cognitive functioning between the study groups. Firstly, as compared to the NC group, the neuropsychological test performance of patients with SIVD was clearly inferior throughout cognitive domains. Secondly, despite comparable levels of global cognitive functioning in the Mini-Mental State Examination, the patients with SIVD had significantly lower scores in executive functioning and delayed memory recall in contrast to the OS patients with larger infarct volume. However, the performance of these two patient groups did not differ in mental speed, short term memory, immediate memory recall, or verbal and visuospatial functions. Of the individual neuropsychological tests, the measures of mental flexibility, response inhibition, verbal fluency, and delayed recall of learned material significantly differentiated the patients with SIVD from the OS patients.

In previous studies, the cognitive performance of patients with variously defined subcortical vascular disease has been compared to healthy elderly subjects ${ }^{62}$ or to patients with

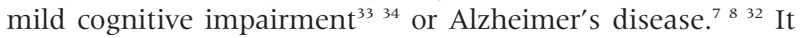
has been suggested that the pattern of cognitive deficits in patients with subcortical ischaemic lesions is distinctly

Table 3 Comparisons of the neuropsychological composite scores of the study groups

\begin{tabular}{|c|c|c|c|c|c|c|c|c|c|c|}
\hline \multirow[b]{3}{*}{ Dependent variable } & \multicolumn{3}{|c|}{ Composite $\mathrm{z}$ scores } & \multicolumn{3}{|c|}{ Between-subjects effects } & \multicolumn{4}{|c|}{ Pairwise contrasts } \\
\hline & \multirow{2}{*}{$\frac{\text { SIVD }}{\text { Mean (SD) }}$} & \multirow{2}{*}{$\frac{\text { OS }}{\text { Mean (SD) }}$} & \multirow{2}{*}{$\frac{\mathrm{NC}}{\text { Mean (SD) }}$} & \multirow[b]{2}{*}{$F$} & \multirow[b]{2}{*}{ p value } & \multirow[b]{2}{*}{$\eta_{p}^{2}$} & \multicolumn{2}{|c|}{ SIVD $v$ OS } & \multicolumn{2}{|c|}{ SIVD v NC } \\
\hline & & & & & & & p value & $\eta_{p}^{2}$ & p value & $\eta_{p}^{2}$ \\
\hline $\begin{array}{l}\text { Speed of mental } \\
\text { processing }\end{array}$ & $-0.15(0.86)$ & $-0.00(0.91)$ & $0.44(0.32)$ & 3.6 & 0.028 & 0.020 & 0.759 & 0.000 & 0.014 & 0.017 \\
\hline Executive functions & $-0.33(0.70)$ & $-0.01(0.66)$ & $0.45(0.42)$ & 14.9 & 0.001 & 0.079 & 0.006 & 0.022 & 0.001 & 0.079 \\
\hline Short term memory & $-0.15(0.76)$ & $0.01(0.87)$ & $0.38(0.88)$ & 3.9 & 0.022 & 0.022 & 0.433 & 0.002 & 0.007 & 0.021 \\
\hline Immediate recall & $-0.25(0.83)$ & $-0.01(0.76)$ & $0.70(0.55)$ & 17.3 & 0.001 & 0.091 & 0.164 & 0.006 & 0.001 & 0.085 \\
\hline Delayed recall & $-0.32(0.87)$ & $-0.00(0.78)$ & $0.74(0.56)$ & 19.3 & 0.001 & 0.100 & 0.028 & 0.014 & 0.001 & 0.098 \\
\hline $\begin{array}{l}\text { Verbal intellectual } \\
\text { functions }\end{array}$ & $-0.23(0.80)$ & $0.02(0.85)$ & $0.53(0.50)$ & 10.1 & 0.001 & 0.055 & 0.248 & 0.004 & 0.001 & 0.052 \\
\hline Visuospatial functions & $-0.32(0.95)$ & $-0.02(0.98)$ & $0.84(0.74)$ & 15.5 & 0.001 & 0.082 & 0.233 & 0.004 & 0.001 & 0.075 \\
\hline
\end{tabular}

MANCOVA F adjusted for age and education. All pairwise comparisons were also significant after Bonferroni correction except for the delayed recall score between SIVD and OS groups. Effect size is expressed as partial eta squared $\left(\eta_{p}{ }^{2}\right)$.

NC, normal control subjects; OS, other stroke patients; SD, standard deviation; SIVD, subcortical ischaemic vascular disease. 
different from that of Alzheimer's disease, ${ }^{78}$ and resembles Parkinson's disease with disproportionate impairment in executive functioning. ${ }^{35}$ However, studies investigating patients with mild cognitive impairment and subcortical vascular disease detected on computed tomography scan have reported diverse results. ${ }^{33}{ }^{34}$ To our knowledge, the present study is the first to describe in detail the cognitive features of SIVD in comparison with elderly stroke patients. Our results indicate that executive deficits, comprising deficiency in mental flexibility, set shifting, response inhibition, and fluency, are salient cognitive characteristics in SIVD. It is notable that verbal fluency can distinguish patients with SIVD not only from those with Alzheimer's disease as suggested earlier, ${ }^{8}$ but also from other elderly stroke patients having large vessel disease and major strokes.

As reported in our previous study, ${ }^{5}$ the patients with SIVD exhibited more depressive symptoms than patients with OS. Subcortical vascular pathology has also been observed to contribute to late life depression in other studies. ${ }^{36}$ According to the vascular depression hypothesis, depressive syndrome in elderly patients can be predisposed, precipitated, or perpetuated by cerebrovascular disease. ${ }^{37}$ In our sample, however, depression scores did not explain the cognitive deficits in patients with SIVD. Although cognitive and mood changes are assumed to be parallel consequences of frontal subcortical disconnections, ${ }^{97}$ our results indicate that they are nevertheless independent processes associated with SIVD.

Another important finding was that controlling for MTA had no effect on the group differences in executive functioning. Instead, the patient groups no longer differed from each other in delayed memory recall after adjusting for MTA. Kramer et $a l^{32}$ have found a heterogeneous pattern of forgetting in patients with SIVD, and they suggest that rapid forgetting in SIVD may imply concomitant Alzheimer's disease. The patients with good retention had worse executive performance and thus were assumed to suffer from pure vascular pathology. Similarly, in our study, it is possible that the delayed memory deficits explained by MTA reflect co-existing Alzheimer pathology, and that only the executive deficits are vascular in origin. Unfortunately, to date there are no means available to unequivocally exclude Alzheimer's disease in vivo and therefore Alzheimer pathology may also contribute to a fraction of our sample in both patient groups. On the other hand, it is not clear whether MTA explicitly indicates Alzheimer's disease or whether it equally belongs to the pathological processes in "pure" SIVD. In a post mortem study, the pattern and the degree of hippocampal neuronal loss were similar in patients with Alzheimer's disease and subcortical vascular dementia. ${ }^{38}$ It has been proposed that hippocampal atrophy in patients with SIVD is a result of a mixture of ischaemic and degenerative processes. ${ }^{39}$ Moreover, in our sample the memory deficits associated with white matter hyperintensities have earlier proven to be mediated by executive deficits and, therefore, they may reflect secondary impairment due to inefficient encoding and retrieval strategies. ${ }^{28}$

It should be noted that the effect sizes of the differences between the two patient groups were rather small. Despite specific MRI findings, there is substantial overlap in the cognitive features of the patients with SIVD and other consecutive stroke patients. The small effect sizes can also reflect high variability in cognitive performance among elderly stroke patients. Notwithstanding this heterogeneity, subtle but significant differences were found between the patient groups. Furthermore, when the patients with SIVD were compared to healthy control subjects, the group differences in cognitive functioning were moderate in magnitude. Another important point concerns the generalisability of the results. They accurately represent the clinical stroke population, but may not be entirely applicable to the general elderly population. Future studies should augment the clinical picture of MRI defined SIVD in population based settings.

The grouping of the neuropsychological test variables into cognitive domains was based on clinical experience and judgment instead of statistical factor modelling. These domains are multidimensional and also, to some extent, overlapping constructs that reflect general, but clinically relevant, aspects of cognitive functioning. In particular, executive functions are here considered as a global concept encompassing a wide array of subfunctions related to attention, volition, planning, and effective performance. ${ }^{21}$ The reliability coefficients indicated that the individual test variables in each of the composite scores had sufficient internal coherence. In addition, the results of the composite and individual scores were consistent with each other.

In conclusion, cognitive deficits in SIVD 3 months post stroke are notable in severity and can be qualitatively distinguished from those of OS patients. The results support the view that SIVD is a separate clinical entity.

\section{ACKNOWLEDGEMENTS}

We thank Pertti Keskivaara, MA for statistical support.

\section{Authors' affiliations}

H Jokinen, H Kalska, Department of Psychology, University of Helsinki, Helsinki, Finland

H Jokinen, R Ylikoski, M Hietanen, Unit of Neuropsychology,

Department of Neurology, Helsinki University Central Hospital, Helsinki, Finland

R Mäntylä, O Salonen, Helsinki Medical Imaging Center, Helsinki, Finland

T Pohjasvaara, Department of Neurology, Lohja Hospital, Helsinki, Finland

T Pohjasvaara, T Erkinjuntti, Memory Research Unit, Department of Neurology, Helsinki University Central Hospital, Helsinki, Finland M Kaste, Department of Neurology, Helsinki University Central Hospital, Helsinki, Finland

This study was financially supported by the Finnish Graduate School of Psychology

Competing interests: none declared

\section{REFERENCES}

1 Bowler JV. The concept of vascular cognitive impairment. J Neurol Sci 2002;203-204:11-15.

2 Erkinjuntti T, Inzitari D, Pantoni L, et al. Limitations of clinical criteria for the diagnosis of vascular dementia in clinical trials. Is a focus on subcortical vascular dementia a solution? Ann N Y Acad Sci 2000;903:262-72.

3 Erkinjuntti T. Subcortical vascular dementia. Cerebrovasc Dis 2002;13/suppl 2):58-60.

4 Erkinjuntti T, Inzitari D, Pantoni L, et al. Research criteria for subcortical vascular dementia in clinical trials. J Neural Transm 2000;59(suppl):23-30

5 Pohjasvaara T, Mäntylä R, Ylikoski R, et al. Clinical features of MRI-defined subcortical vascular disease. Alzheimer Dis Assoc Disord 2003;17:236-42.

6 Kramer JH, Reed BR, Mungas D, et al. Executive dysfunction in subcortical ischemic vascular disease. I Neurol Neurosurg Psychiatry 2002;72:217-20.

7 Schmidtke K, Hüll M. Neuropsychological differentiation of small vessel disease, Alzheimer's disease and mixed dementia. J Neurol Sci 2002;203204:17-22.

8 Tierney MC, Black SE, Szalai JP, et al. Recognition memory and verbal fluency differentiate probable Alzheimer disease from subcortical ischemic vascular dementia. Arch Neurol 2001;58:1654-9.

9 Cummings JL. Frontal-subcortical circuits and human behavior. Arch Neurol 1993;50:873-80

10 Pugh KG, Lipsitz LA. The microvascular frontal-subcortical syndrome of aging. Neurobiol Aging 2002;23:421-31.

11 Román GC, Erkinjuntti T, Wallin A, et al. Subcortical ischaemic vascular dementia. Lancet Neurol 2002;1:426-36.

12 Pohjasvaara T, Erkinjuntti T, Vataja R, et al. Dementia three months after stroke. Baseline frequency and effect of different definitions of dementia in the Helsinki Stroke Aging Memory Study (SAM) cohort. Stroke 1997; 28:785-92.

13 Pohjasvaara T, Erkinjuntti T, Ylikoski R, et al. Clinical determinants of poststroke dementia. Stroke 1998;29:75-81.

14 Folstein MF, Folstein SE, McHugh PR. 'Mini-mental state': a practical method for grading the cognitive state of patients for the clinician. $J$ Psychiatr Res 1975; 12:189-98. 
15 Ylikoski R, Ylikoski A, Erkinjuntti T, et al. White matter changes in healthy elderly persons correlate with attention and speed of mental processing. Arch Neurol 1993;50:818-24.

16 Leskelä $M$, Hietanen $M$, Kalska $H$, et al. Executive functions and speed of mental processing in elderly patients with frontal or nonfrontal ischemic stroke. Eur J Neurol 1999;6:653-61.

17 Jokinen $\mathbf{H}$, Kalska H, Ylikoski R, et al. Medial temporal lobe atrophy and memory deficits in elderly stroke patients. Eur J Neurol 2004; 11:825-32.

18 Reitan RM. Validity of the Trail Making Test as an indicator of organic brain damage. Percept Mot Skills 1958:8:271-6.

19 Perret E. The left frontal lobe of man and the suppression of habitual responses in verbal categorical behavior. Neuropsychologia 1974;12:323-30.

20 Nelson HE. A modified card sorting test sensitive to frontal lobe defects. Cortex 1976;12:313-24.

21 Lezak MD, Howieson DB, Loring DW. Neuropsychological assessment, 4th ed. New York: Oxford University Press, 2004.

22 Wechsler D. A standardized memory scale for clinical use. J Psychol 1945; 19:87-95.

23 Fuld PA. Fuld object memory evaluation. Chicago: Stoelting, 1982

24 Wechsler D. WMS-R, Wechsler memory scale-revised manual. San Antonio, TX: Psychological Corporation, 1987

25 Wechsler D. WAIS-R manual. New York: Psychological Corporation, 1981

26 Mäntylä R, Erkinjuntti T, Salonen O, et al. Variable agreement between visual rating scales for white matter hyperintensities on MRI. Stroke 1997; 28:1614-23.

27 Mäntylä R, Pohjasvaara T, Vataja R, et al. MRI pontine hyperintensity after supratentorial ischemic stroke relates to poor clinical outcome. Stroke 2000;31:695-700.

28 Jokinen $H$, Kalska $H$, Mäntylä R, et al. White matter hyperintensities as a predictor of neuropsychological deficits post-stroke. J Neurol Neurosurg Psychiatry (in press).
29 Cohen J. Statistical power analysis for the behavioral sciences. Hillsdale, NJ: Lawrence Erlbaum, 1988.

30 American Psychiatric Association Committee on Nomenclature and Statistics. Diagnostic and statistical manual of mental disorders (DSM-III-R), 3rd ed., revised. Washington, DC: American Psychiatric Association, 1987

31 Beck AT, Ward CH, Mendelson M, et al. An inventory for measuring depression. Arch Gen Psychiatry 1961:4:561-71.

32 Kramer JH, Mungas D, Reed BR, et al. Forgetting in dementia with and without subcortical lacunes. Clin Neuropsychol 2004; 18:32-40.

33 de Mendoca A, Ribeiro F, Guerreiro M, et al. Clinical significance of subcortical vascular disease in patients with mild cognitive impairment. Eur J Neurol 2005; 12:125-30.

34 Galluzzi S, Sheu C-F, Zanetti O, et al. Distinctive clinical features of mild cognitive impairment with subcortical cerebrovascular disease. Dement Geriatr Cogn Disord 2005; 19:196-203.

35 Libon DJ, Bogdanoff B, Leopold N, et al. Neuropsychological profiles associated with subcortical white matter alterations and Parkinson's disease: implications for the diagnosis of dementia. Arch Clin Neuropsychol 2001;16:19-32.

36 Krishnan KRR, Taylor WD, McQuoid DR, et al. Clinical characteristics of magnetic resonance imaging-defined subcortical ischemic depression. Biol Psychiatry 2004;55:390-7.

37 Alexopoulos GS, Meyers BS, Young RC, et al. 'Vascular depression' hypothesis. Arch Gen Psychiatry 1997;54:915-22.

38 Kril JJ, Patel S, Harding AJ, et al. Patients with vascular dementia due to microvascular pathology have significant hippocampal neuronal loss. J Neurol Neurosurg Psychiatry 2002;72:747-51.

39 Fein G, Di Sclafani V, Tabane J, et al. Hippocampal and cortical atrophy predict dementia in subcortical ischemic vascular disease. Neurology 2000;55:1626-35 